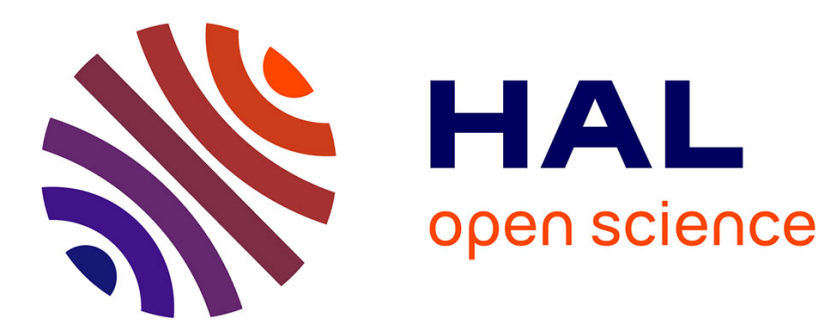

\title{
Periodic unit cell reconstruction of porous media: Application to open-cell aluminum foams
}

\author{
C. Perrot, R. Panneton, X. Olny
}

\section{To cite this version:}

C. Perrot, R. Panneton, X. Olny. Periodic unit cell reconstruction of porous media: Application to open-cell aluminum foams. Journal of Applied Physics, 2007, 101 (11), pp.113538-11. 10.1063/1.2745095 . hal-00731850

\section{HAL Id: hal-00731850 \\ https://hal.science/hal-00731850}

Submitted on 17 Sep 2012

HAL is a multi-disciplinary open access archive for the deposit and dissemination of scientific research documents, whether they are published or not. The documents may come from teaching and research institutions in France or abroad, or from public or private research centers.
L'archive ouverte pluridisciplinaire HAL, est destinée au dépôt et à la diffusion de documents scientifiques de niveau recherche, publiés ou non, émanant des établissements d'enseignement et de recherche français ou étrangers, des laboratoires publics ou privés. 


\title{
Periodic unit cell reconstruction of porous media: Application to open-cell aluminum foams
}

\author{
Camille Perrot ${ }^{\mathrm{a})}$ \\ GAUS, Department of Mechanical Engineering, Université de Sherbrooke (Qc), Quebec J1K 2R1, Canada \\ and ENTPE, DGCB URA CNRS 1652, rue Maurice Audin, 69518 Vaulx en Velin, France
}

Raymond Panneton

GAUS, Department of Mechanical Engineering, Université de Sherbrooke (Qc), Quebec J1K 2R1, Canada

\begin{abstract}
Xavier Olny
Département Villes et Territoires/Groupe AUE, Centre d'Études Techniques de l'Équipement de Lyon, 46 rue Saint Théobald-Boîte Postale 128, 38081 L'isle d'Abeau Cedex, France
\end{abstract}

(Received 6 February 2007; accepted 19 April 2007; published online 15 June 2007)

\begin{abstract}
In this article, the issue of reconstructing an idealized periodic unit cell (PUC) to represent a porous medium is examined by means of microcomputed tomography $(\mu \mathrm{CT})$. Using $\mu \mathrm{CT}$, three-dimensional images of open-cell foam are collected and used to characterize the representative parameters of its cellular morphology. These parameters are used in order to reconstruct the porous medium by means of an idealized PUC: a tetrakaidecahedron with ligaments of triangular cross sections, whose characteristic dimensions have been measured on the $\mu \mathrm{CT}$ images. The proposed reconstruction of the idealized PUC is applied to four aluminum foams. The averaged macroscopic properties of the foams (open porosity and thermal characteristic length) are deduced from their respective PUC model and compared to experimental measurements and literature data. Good correlations are obtained. For each of the foams, this provides a parameterized idealized periodic unit cell on which the partial differential equations governing sound dissipation and propagation in foams can be solved with a view to studying the microphysical basis of the acoustical macrobehavior. @ 2007 American Institute of Physics. [DOI: 10.1063/1.2745095]
\end{abstract}

\section{INTRODUCTION}

Most porous media possess a very complex internal structure which is difficult to grasp quantitatively. However, it would be desirable to be able to quantify its local geometry by an idealized periodic unit cell (PUC) whose characteristic parameters would be directly measured on three-dimensional images obtained from microcomputed tomography $(\mu \mathrm{CT})$. Apart from the intrinsic need for characterizing the cell morphology itself, insight into the morphology of an idealized PUC would be helpful for understanding the microphysical basis behind transport phenomena, such as the propagation and absorption properties of acoustical waves through a porous medium. ${ }^{1,2}$ In addition, an idealized PUC-which is parameterized by nature - could be used to optimize the cellular structure of a porous medium from a bottom-up approach to maximize sound proofing. The major purpose of this article deals with the characterization of the internal structure of open-cell foams and its reconstruction using the concept of the idealized periodic unit cell.

The appropriate theoretical framework of this work is - of course - cellular solids, and a very readable textbook of the most interesting quantities and concepts is given by Gibson and Ashby. ${ }^{3}$ The text by Weaire and Hutzler ${ }^{4}$ also provides the reader with an introduction to the physics of foams and the most advanced tools to model high porosity

\footnotetext{
a) Author to whom correspondence should be addressed; electronic mail: camille.perrot@usherbrooke.ca
}

open-cell foams such as the surface evolver program. ${ }^{5} \mathrm{Sev}$ eral authors have reported experimental studies of local geometry properties of fully saturated high porosity open-cell metal foams. ${ }^{6-9}$ Zhou et al. ${ }^{6}$ have investigated the morphology of foams from a mechanical property analysis viewpoint. They presented principal cell diameter, ligament thickness, and ligament length data for three combinations of porosity and pore density. However, this was done using a destructive metallographic examination technique based on optical microscopy and stereological image analysis. A nondestructive method for making three-dimensional geometric measurements is x-ray $\mu \mathrm{CT}$. This technique has been used in several investigations to obtain three-dimensional images of the internal structure of foams. In these investigations, cell wall thickness and cell size distribution were the parameters of interest. Montminy et al. ${ }^{7}$ have developed an algorithm for finding the nodal centers and ligament lengths on $\mu \mathrm{CT}$ images of open-cell polymer foams; however, they did not provide the ligament thicknesses. Scheffler et al. ${ }^{8}$ have characterized an open-cell aluminum foam sample for use as a zeolite substrate. They presented ligament thickness and cell diameter distributions for this foam sample without giving ligament lengths. Schmierer et al. ${ }^{9}$ have used $\mu \mathrm{CT}$ to determine the thickness distribution of the ligaments of open-cell metal foams and their surface area for four combinations of porosity and pore density.

Similarly to the previous works, the present work focuses on the characterization of the representative morphological parameters (number of faces/cell, number of edges/ 
face, degree of anisotropy, cell diameter, cell ligament thickness, cell ligament length) of the cells forming a high porosity open-cell foam. Contrary to previous works, this time all the local parameters are presented simultaneously and obtained using a nondestructive method by direct threedimensional measurements performed at the cell size level. The characterized parameters are used to build an idealized PUC. The purely geometrical macroscopic properties-open porosity and thermal characteristic length (Appendix A)—of the idealized reconstructed PUC are then determined by spatial integration and compared with experimental measurements and literature data with a view to validating the proposed idealized PUC reconstruction approach. It is worth mentioning that the studied thermal characteristic lengthcommonly used in acoustics of porous media-is a parameter closely related to the specific surface of the solid porous frame. ${ }^{10}$

This article is organized as follows. Section II describes the studied porous media, the experimental setup, and the method of idealized PUC reconstruction. Section III presents the measured cellular parameters, the purely geometrical macroscopic parameters of the reconstructed unit cells, and compares these results to experimental measurements and literature data. Finally, the main conclusions to this work are presented in Sec. IV.

\section{MATERIALS AND METHODS}

\section{A. Studied open-cell aluminum foams}

In this article, ERG Duocel open-cell aluminum foams (produced by ERG, Oakland, CA) are studied. Foams with four levels of pore sizes $[10,20$, and 40 pores per inches (ppi)] are investigated. The value for the pore density is the manufacturer's designation — not necessarily an indication of true pore size. The foam material is aluminum 6101 alloy, which consists (in wt \%) of $99.32 \mathrm{Al}, 0.19 \mathrm{Mg}, 0.27 \mathrm{Si}, 0.12$ $\mathrm{Fe}$, and 0.1 others $(\mathrm{Cu}, \mathrm{Mn}, \mathrm{Zn}, \mathrm{B})$. ERG Duocel open-cell aluminum foams are fabricated following a process similar to investment casting, which replicates the porous preform of a polymer precursor. ${ }^{11-13}$ Foam specimens are machined from the as-received large foam blocks supplied by ERG. In an effort to avoid possible effects of plastic deformation due to diamond sawing, cylindrical samples of 10 and $20 \mathrm{~mm}$ in diameter were obtained via electrodischarge machining.

\section{B. $\mu \mathrm{CT}$}

Unlike synchrotron radiations generated by relativistic electrons or positrons circulating on a bent trajectory, x-ray laboratory sources are based on a polychromatic standard x-ray tube. The polychromaticity of the beam generates beam "hardening," leading to unavoidable artifacts in tomographic images. The artifact derives its name from its underlying cause - the increase in mean $\mathrm{x}$-ray energy or hardening of the X-ray beam as it passes through the scanned object. Because lower-energy $\mathrm{x}$ rays are attenuated more readily than higher-energy $\mathrm{x}$ rays, a polychromatic beam passing through an object preferentially loses the lower energy part of its spectrum. The end result is a beam that, though diminished in overall intensity, has a higher average energy than

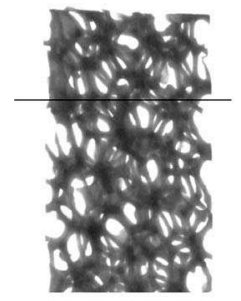

a)

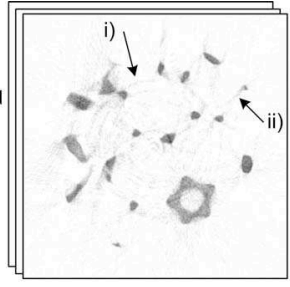

b)

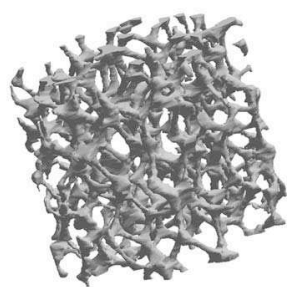

c)
FIG. 1. Main steps of the reconstruction process by x-ray $\mu \mathrm{CT}$. (a) Acquisition of classical radiographies, or "x-ray shadow images" of the real sample. (b) Reconstruction of bidimensional cross sections of the real sample showing (i) "ring artifacts" and (ii) "starburst artifacts." (c) Reconstruction of the three-dimensional image of an axial portion of the real sample, after binarisation of the two-dimensional images.

the incident beam. This also means that, as the beam passes through an object, the effective attenuation coefficient of any material diminishes, thus making short ray paths proportionally more attenuating that long ray passes. In x-ray $\mu \mathrm{CT}$ images of sufficiently attenuating materials, this process generally manifests itself as an artificial darkening at the center of long ray-paths and a corresponding brightening near the edges. Beam hardening can be a pernicious artifact because it changes the linear absorption coefficient value of a material (or gas) depending upon its location in an image. Thus, the attempt to utilize a single linear absorption coefficient range to identify a particular material may become problematic.

The local geometry of the foam samples are obtained using an x-ray $\mu \mathrm{CT}$ desktop ( $\mu \mathrm{CT} 1072$ system, Skyscan, Aartselaar, Belgium) available at the Université de Sherbrooke. A microfocus x-ray tube with an $8 \mu \mathrm{m}$ focal spot size operates at $20-80 \mathrm{kV} / 100 \mu \mathrm{A}$ current and generates a divergent polychromatic beam. The sample is mounted on a step-by-step rotating sample holder, including translations to position and rotate it in the beam. A two-dimensional detector records the beam transmitted through the sample. The two dimensional detector is based on a charged coupled device (CCD) coupled to a scintillator screen. It includes a $1024 \times 1024$ CCD chip and offers a 4096 brightness gradation. The magnification changes correspondingly to the mechanical movement of the object from detector to the tube.

\section{Microstructure acquisition}

\section{Acquisition of radiographies}

The four open-cell aluminum foams corresponding to different pore densities are $\mathrm{x}$ rayed using the 1072 Skyscan $\mathrm{x}$-ray $\mu \mathrm{CT}$ desktop. A good contrast is found between the fluid (air) and solid (aluminum) phases of the sample when the $\mu \mathrm{CT}$ is operating at $80 \mathrm{kV}$ and $62 \mu \mathrm{A}$, with an exposure time of $112 \mathrm{~ms}$. A pixel size of $21.8 \mu \mathrm{m}$ is used with the minimum object magnification of 10. During acquisition, the sample is sequentially rotated over a total angular range of $180^{\circ}$. Typically, 200 equiangular radiographic images per tested foam sample are acquired (angular step: $0.9^{\circ}$ ). In addition, dark current and reference images are recorded with the same exposure time to perform flat field correction, Fig. 1(a). This correction removes inhomogeneities from the $\mathrm{x}$-ray field and detector response. 


\section{Reconstruction of cross sections}

The set of acquired $\mathrm{x}$-ray images is processed through a modified Feldkamp cone-beam reconstruction algorithm ${ }^{14}$ provided with the $\mu \mathrm{CT}$ Skyscan system to get the twodimensional reconstructed images of the sample. The reconstructed cross-section images represent the two-dimensional maps of the linear absorption coefficient $\mu(x, y)$ for varying elevation $z$. The thickness of the reconstructed cross sections is fixed by the pixel size, being $21.8 \mu \mathrm{m}$. A typical slice of the sample after reconstruction is shown at Fig. 1(b) for the 40 ppi aluminum foam. Two kinds of artifacts are visible on this image: (i) ring artifacts and (ii) starburst artifacts. Ring artifacts are appearing as full or partial circles centered on the rotational axis. They are caused by differential detector sensitivity to varying beam hardness (mean x-ray energy). Starburst artifacts can form in bright streaks emanating from the object for a short distance into nearby material. They can arise if the scanned material includes features that are of much higher density than the surrounding medium. All of these artifacts have a common origin: the nonmonochromaticity of the incident x-rays beam. For a full discussion of these artifacts, see Ref. 15. A serial reconstruction of cross sections is finally used after segmentation and averaging of initial cross sections for the reconstruction of a complete three-dimensional object, Fig. 1(c).

\section{Reconstruction of three-dimensional objects}

a. Smoothing and segmentation based on visual criterions-

Due to the reconstruction artifacts, an artificial roughness is appearing at the surface of the reconstructed objects. A smoothing of the reconstructed cross sections is then achieved by means of a $4 \times 4$ averaging filter (with TConv, software provided with the Skyscan system). As a counterpart, the resolution of the reconstructed objects is divided by 4, leading to a pixel size of $87.2 \mu \mathrm{m}$. We shall see later on that the mean thickness value of the thinnest ligament is approximately $370 \mu \mathrm{m}$. With a spatial resolution of $87.2 \mu \mathrm{m} / \mathrm{pixel}$, the height of a ligament's triangular cross section is composed of 4.24 pixels in average. For a measurement uncertainty of \pm 1 pixel, the mean thickness of the thinnest ligament's cross section yields $4.24 \pm 1$ pixels. It leads to a variation coefficient of approximately $23 \%$ (standard deviation/mean) on the measurements. In practice, this large uncertainty on the measurements does not allow to estimate conveniently the purely geometrical macroscopic parameters of the foam (open porosity and thermal characteristic length) by direct spatial integration on the volume and surface elements of the three-dimensional $\mu \mathrm{CT}$ reconstructed object. In order to obtain an estimation of the area of a digitized circle with less than $1 \%$ of error, a number of 15 pixels/ diameter is generally required. ${ }^{16,17}$ To overcome this difficulty, and taking into account the limited resolution of the apparatus, a continuum disk may be associated to each digitized disk, leading to a kind of continuous idealization of the medium. That is the idea which will be applied throughout this article.

After the smoothing of the reconstructed cross sections, the key point is image segmentation for separating the solid phase (aluminum) from the fluid phase (air). For this pur-
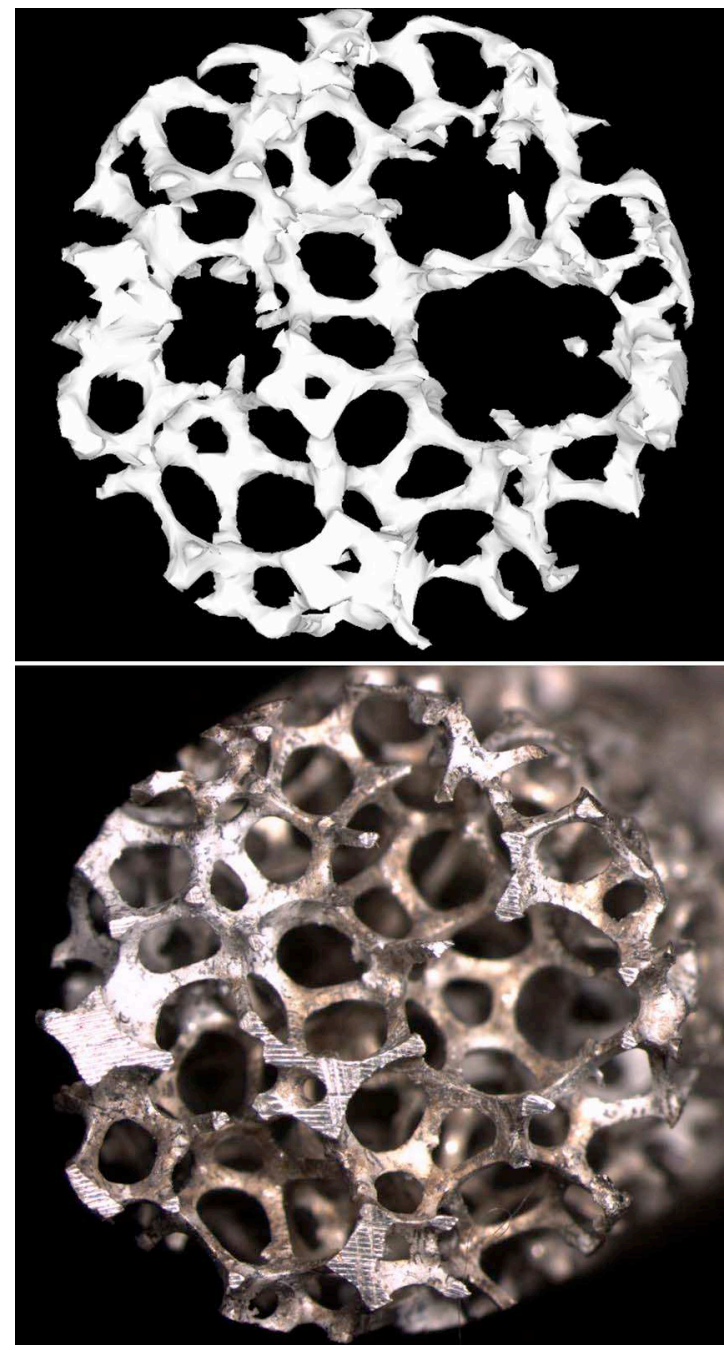

FIG. 2. Segmentation found manually by visual comparison of a superficial section of the segmented volume (top) and an optical micrograph of the same superficial section of the real sample (bottom).

pose, a global threshold is applied: pixels having a gray level superior (respectively inferior) to a given threshold value are affected to the solid phase (respectively fluid) with binary level of 1 (respectively zero) in the resulting image. The threshold set was found manually by visual comparison of a superficial reconstructed section of the segmented volume, Fig. 2 (top) and an optical micrograph of the same superficial section of the real sample, Fig. 2 (bottom). Furthermore, an example of quantitative comparison is depicted in Fig. 3, where the characteristic dimensions of a typical pattern identified on the reconstructed and original superficial sections are compared with good accuracy. In practice, the distances of the digitized objects are effectively conserved, which allows us to proceed with the idealization of the medium.

b. Reconstruction of isolated cells Once the reconstructed cross sections have been smoothed, and the threshold adequately determined, isolated cells can be reconstructed. From the set of reconstructed cross sections, it is possible to determine a region of interest containing only one cell, and to identify the high and low limits from which a threedimensional cell will be reconstructed. This process is repeated to collect a sufficient number of cells for each of the foams to characterize, Fig. 4. 

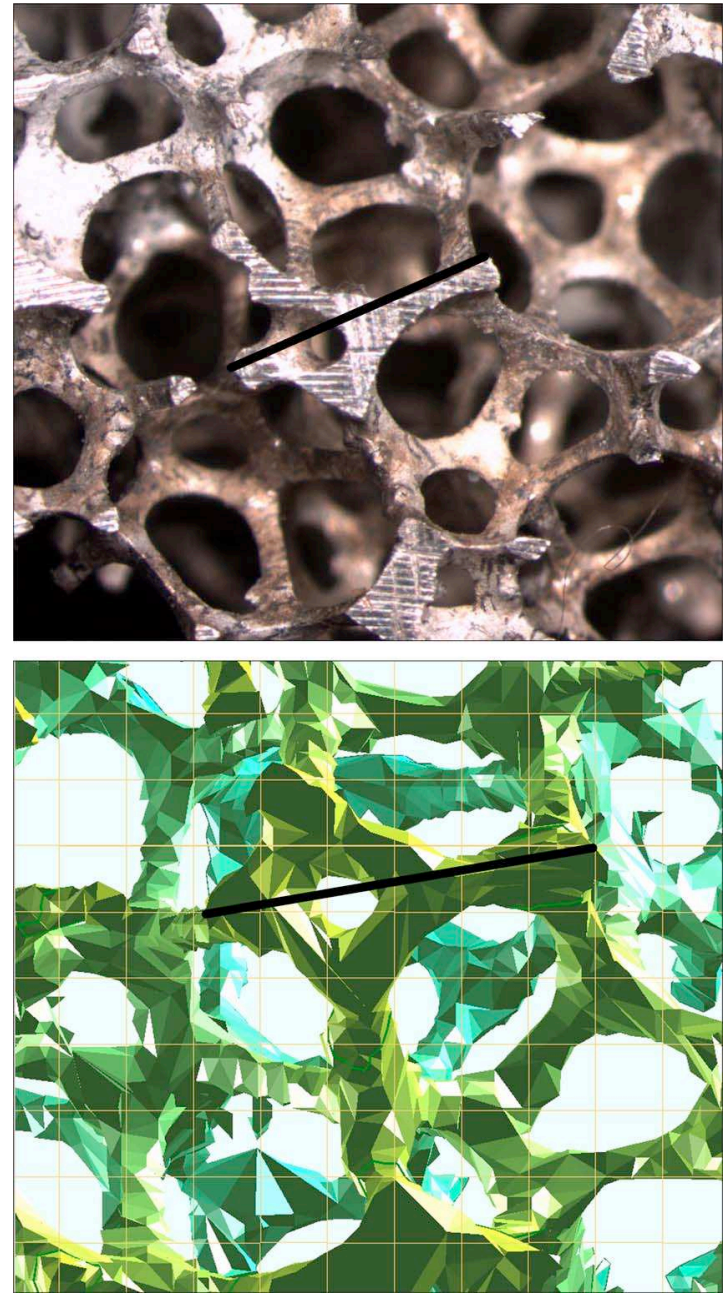

FIG. 3. Details of a quantitative comparison between a superficial section of the segmented volume (bottom) and a micrograph of the same superficial section of the real sample (top). The distance measured on the reconstructed object is $2743 \mu \mathrm{m}$. The distance measured on the reference object is $2780 \mu \mathrm{m}$. The relative error made on the measured distance of the reconstructed object is approximately $1.3 \%$.

\section{Measurement of the cell morphological parameters}

The next step is the measurement of the morphological parameters of the $\mathrm{x}$-ray $\mu \mathrm{CT}$ reconstructed cells. To charac-

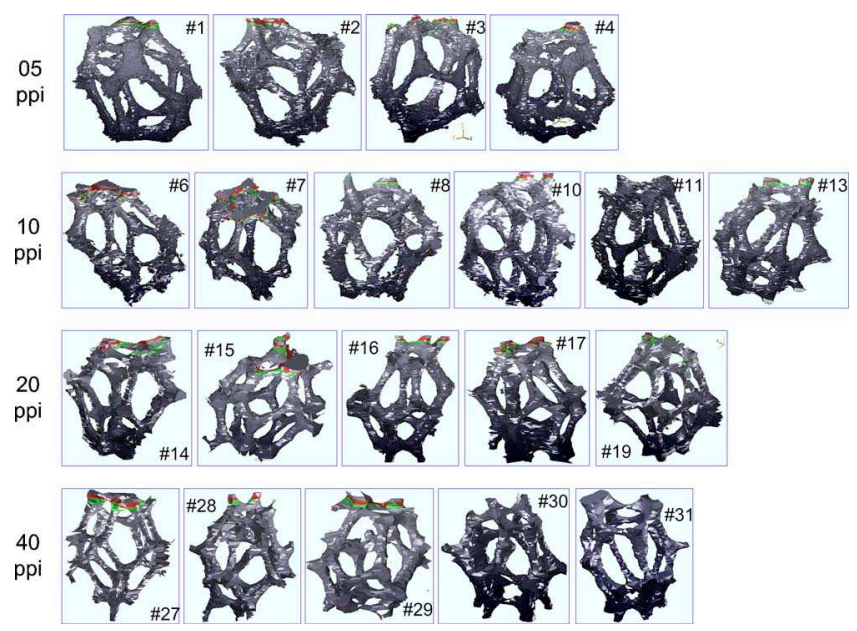

FIG. 4. Three-dimensional reconstructed cells for the four ERG open-cell aluminum foams.
TABLE I. Statistics on the measured parameters characterizing the cellular shape of the four ERG open-cell aluminum foams (mean \pm standard deviation). The parameters are directly measured on the corresponding threedimensional $\mu \mathrm{CT}$ images of the reconstructed cells shown in Fig. 4.

\begin{tabular}{cccc}
\hline \hline $\begin{array}{c}\text { Pore density } \\
\text { (ppi) }\end{array}$ & Faces/cell $f$ & Edges/face $n$ & Degree of anisotropy DA \\
\hline 5 & $12.00 \pm 1.41$ & $5.13 \pm 0.11$ & $1.26 \pm 0.18$ \\
10 & $12.83 \pm 1.72$ & $5.19 \pm 0.10$ & $1.56 \pm 0.20$ \\
20 & $12.40 \pm 1.14$ & $5.06 \pm 0.11$ & $1.42 \pm 0.12$ \\
40 & $12.60 \pm 0.89$ & $5.17 \pm 0.14$ & $1.42 \pm 0.08$ \\
Overall 5-40 & $12.46 \pm 1.28$ & $5.14 \pm 0.12$ & $1.41 \pm 0.18$ \\
\hline \hline
\end{tabular}

terize the morphology of an isolated cell, the following parameters have to be measured: the number of faces/cell, the number of edges/face, the degree of anisotropy, the smallest and largest characteristic radii of the cell, and the length and thickness of the ligaments. Appendix B gives the details on how and where these parameters have to be measured on a reconstructed cell. All these parameters provide a detailed snapshot of the foam's microstructure, and are used to reconstruct its idealized PUC representation.

\section{RESULTS AND DISCUSSION}

\section{A. Quantitative analysis of the three-dimensional cellular morphology}

In the context of this work, the parameters characterizing the shape of the cells are the number of faces per cell $f$, the number of edges per face $n$, and the degree of anisotropy DA-see Appendix B. They are directly measured on the $\mu \mathrm{CT}$ three-dimensional images of the reconstructed cells shown in Fig. 4. The statistics (mean \pm standard deviation) on the measured parameters are presented in Table I for the four studied ERG open-cell aluminum foams (5, 10, 20, and $40 \mathrm{ppi})$. One can observe that the shape parameters are statistically independent from the pore density. The overall statistics on the analyzed cells yield $f=12.46 \pm 1.28$ faces/cell, $n=5.14 \pm 0.12$ edges/face, and a degree of anisotropy DA of $1.41 \pm 0.18$. The degree of anisotropy describes the elongation of the cells in the rise direction versus that in the other principal directions. Here, $\mathrm{DA}=1.41$ reveals a rather elongated structure, closer to the shape of an egg than a sphere. Theoretical models of periodic unit-cells such as the Kelvin's tetrakaidecahedron $\quad(f=14$ faces/cell, $n=5.14$ edges $/$ face $)$ (Ref. 18) and the Weaire-Phelan partition $(f=13.5$ faces/cell, $n=5.11$ edges/face) (Ref. 19) suggest that these shapes, once elongated, may be proposed as an acceptable approximation of the studied shapes, see Table II. However,

TABLE II. Main idealized periodic unit cells of monodisperse foams.

\begin{tabular}{lcc}
\hline \hline & Kelvin $^{\mathrm{a}}$ & Weaire-Phelan $^{\mathrm{b}}$ \\
\hline Faces/cell, $f$ & 14 & 13.5 \\
Edges/face, $n$ & 5.14 & 5.11 \\
\hline \hline
\end{tabular}

${ }^{\mathrm{a}}$ Reference 18 .

${ }^{\mathrm{b}}$ Reference 19. 
TABLE III. Statistics (mean and variation coefficient) on the measured parameters characterizing the cellular size of the four ERG open-cell aluminum foams. The parameters are directly measured on the corresponding three-dimensional $\mu \mathrm{CT}$ images of the reconstructed cells shown in Fig. 4. The variation coefficient VC is defined as the standard deviation to mean ratio.

\begin{tabular}{|c|c|c|c|c|c|c|c|}
\hline \multirow[b]{2}{*}{$\begin{array}{l}\text { Pore density } \\
\text { (ppi) }\end{array}$} & \multirow[b]{2}{*}{ Cell No. } & \multicolumn{2}{|c|}{ Principal characteristic radius } & \multicolumn{2}{|c|}{ Ligament length, $l$} & \multicolumn{2}{|c|}{ Ligament thickness, $t$} \\
\hline & & $\begin{array}{c}R_{1} \\
(\mathrm{~mm}) \\
\end{array}$ & $\begin{array}{c}R_{3} \\
(\mathrm{~mm})\end{array}$ & $\begin{array}{l}\text { Mean } \\
(\mathrm{mm})\end{array}$ & $\begin{array}{l}\text { VC } \\
(\%) \\
\end{array}$ & $\begin{array}{l}\text { Mean } \\
(\mathrm{mm}) \\
\end{array}$ & $\begin{array}{l}\mathrm{VC} \\
(\%) \\
\end{array}$ \\
\hline \multirow[t]{6}{*}{05} & 1 & 2.85 & 3.82 & 2.40 & 31.16 & 0.77 & 21.88 \\
\hline & 2 & 2.49 & 2.54 & 2.21 & 29.59 & 0.78 & 21.40 \\
\hline & 3 & 2.43 & 3.53 & 2.28 & 33.20 & 0.73 & 16.74 \\
\hline & 4 & 2.62 & 3.19 & 2.32 & 27.01 & 0.76 & 15.47 \\
\hline & Mean & 2.60 & 3.27 & 2.30 & 30.24 & 0.76 & 18.92 \\
\hline & $\mathrm{VC}(\%)$ & 7.11 & 16.79 & 3.38 & & 2.82 & \\
\hline \multirow[t]{8}{*}{10} & 6 & 2.22 & 4.25 & 1.89 & 31.53 & 0.64 & 20.39 \\
\hline & 7 & 2.03 & 2.84 & 1.74 & 32.59 & 0.69 & 30.11 \\
\hline & 8 & 2.12 & 3.24 & 1.97 & 30.79 & 0.60 & 19.45 \\
\hline & 10 & 2.05 & 3.34 & 1.63 & 28.08 & 0.66 & 19.39 \\
\hline & 11 & 2.09 & 3.24 & 1.91 & 37.53 & 0.63 & 18.09 \\
\hline & 13 & 2.11 & 2.82 & 1.83 & 27.42 & 0.64 & 37.11 \\
\hline & Mean & 2.10 & 3.29 & 1.83 & 31.41 & 0.64 & 24.21 \\
\hline & $\mathrm{VC}(\%)$ & 3.29 & 15.80 & 6.87 & & 4.79 & \\
\hline \multirow[t]{7}{*}{20} & 14 & 1.71 & 2.50 & 1.53 & 29.32 & 0.45 & 19.56 \\
\hline & 15 & 1.66 & 2.32 & 1.43 & 26.50 & 0.45 & 23.37 \\
\hline & 16 & 1.47 & 2.20 & 1.28 & 34.59 & 0.46 & 18.04 \\
\hline & 17 & 1.68 & 2.05 & 1.41 & 24.49 & 0.46 & 24.04 \\
\hline & 19 & 1.66 & 2.54 & 1.50 & 41.84 & 0.48 & 29.38 \\
\hline & Mean & 1.63 & 2.32 & 1.43 & 31.36 & 0.46 & 22.94 \\
\hline & $\mathrm{VC}(\%)$ & 5.83 & 8.83 & 6.88 & & 2.75 & \\
\hline \multirow[t]{7}{*}{40} & 27 & 1.42 & 2.05 & 1.25 & 30.82 & 0.35 & 24.08 \\
\hline & 28 & 1.39 & 2.12 & 1.22 & 32.85 & 0.40 & 21.08 \\
\hline & 29 & 1.32 & 1.72 & 1.21 & 26.44 & 0.34 & 15.92 \\
\hline & 30 & 1.44 & 1.97 & 1.18 & 32.30 & 0.39 & 15.53 \\
\hline & 31 & 1.36 & 1.98 & 1.06 & 36.93 & 0.39 & 26.30 \\
\hline & Mean & 1.39 & 1.97 & 1.19 & 31.73 & 0.37 & 20.64 \\
\hline & $\mathrm{VC}(\%)$ & 3.42 & 7.71 & 6.20 & & 6.92 & \\
\hline
\end{tabular}

note that the diversity of the observed cellular shapes shows these models to be simplified structures of real open cell foams. $^{20,21}$

Similar to the previous identification, measurements at the cell-size level allow the identification of the characteristic lengths of a representative unit-cell candidate. In the context of this work, the measured characteristic lengths are the ligament length $l$, the ligament thickness $t$, the smallest cell radius $R_{1}$, and the largest cell radius $R_{3}$. Their measurements are discussed in Appendix B. Results of the measured parameters are presented in Table III. One can note that all the characteristic parameters decrease as the pore density increases, and not in a linear way. However, a closer look at the results shows that the ligament length to thickness ratio $(l / t)$, the small radius to ligament length ratio $\left(R_{1} / l\right)$, and the large to small radius ratio $\left(R_{3} / R_{1}=\mathrm{DA}\right)$ are statistically identical for the four pore densities-see Table IV. This observation indicates that from one pore density to another, the geometric configuration of the cells is unaltered and their size parameters are only magnified by a given factor.

While only one measurement was sufficient to obtain the minimum and maximum radius of a unit cell, approximately
36 measurements were required to estimate its ligament length and thickness. As observed in Table III, the variation coefficient on individual cell measurements is rather large $(\mathrm{VC} \approx 30 \%$ for ligament length and $\mathrm{VC} \approx 20 \%$ for ligament thickness). To better capture the meaning of these variations, Fig. 5 shows the distribution of the ligament length and thickness for each of the four aluminum foams. The dark circles point to the mean values given in Table III. As one can note, the ligament length generally follows a bimodal distribution and the ligaments thickness a rather monomodal right-skewed distribution typical to statistics describing natu-

TABLE IV. Ratios between some of the cellular size parameters of the four ERG open-cell aluminum foams given in Table III (mean \pm standard deviation).

\begin{tabular}{cccc}
\hline \hline $\begin{array}{c}\text { Pore density } \\
(\mathrm{ppi})\end{array}$ & $l / t$ & $R_{1} / l$ & $R_{3} / R_{1}=\mathrm{DA}$ \\
\hline 05 & $3.03 \pm 0.14$ & $1.13 \pm 0.05$ & $1.26 \pm 0.18$ \\
10 & $2.85 \pm 0.31$ & $1.15 \pm 0.07$ & $1.56 \pm 0.20$ \\
20 & $3.12 \pm 0.24$ & $1.14 \pm 0.03$ & $1.42 \pm 0.12$ \\
40 & $3.18 \pm 0.36$ & $1.17 \pm 0.08$ & $1.42 \pm 0.08$ \\
\hline \hline
\end{tabular}



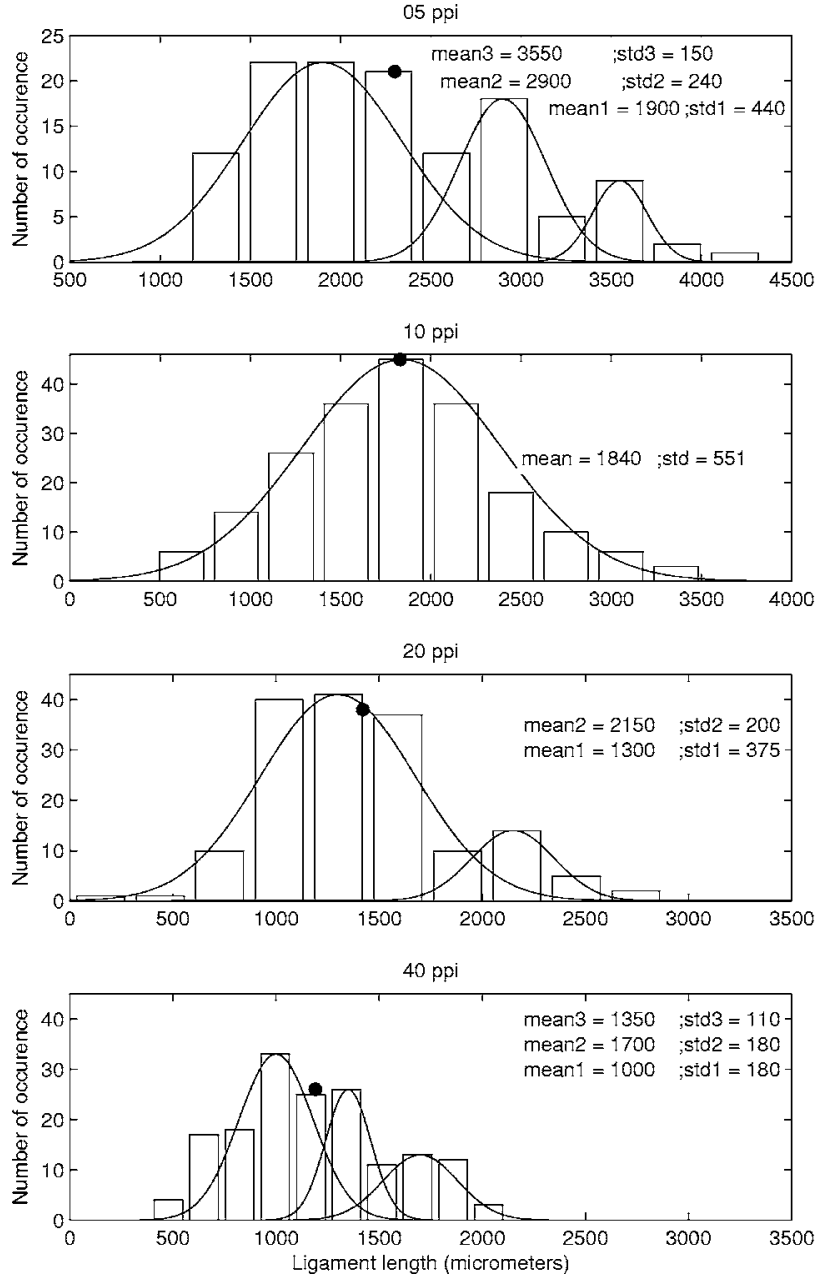
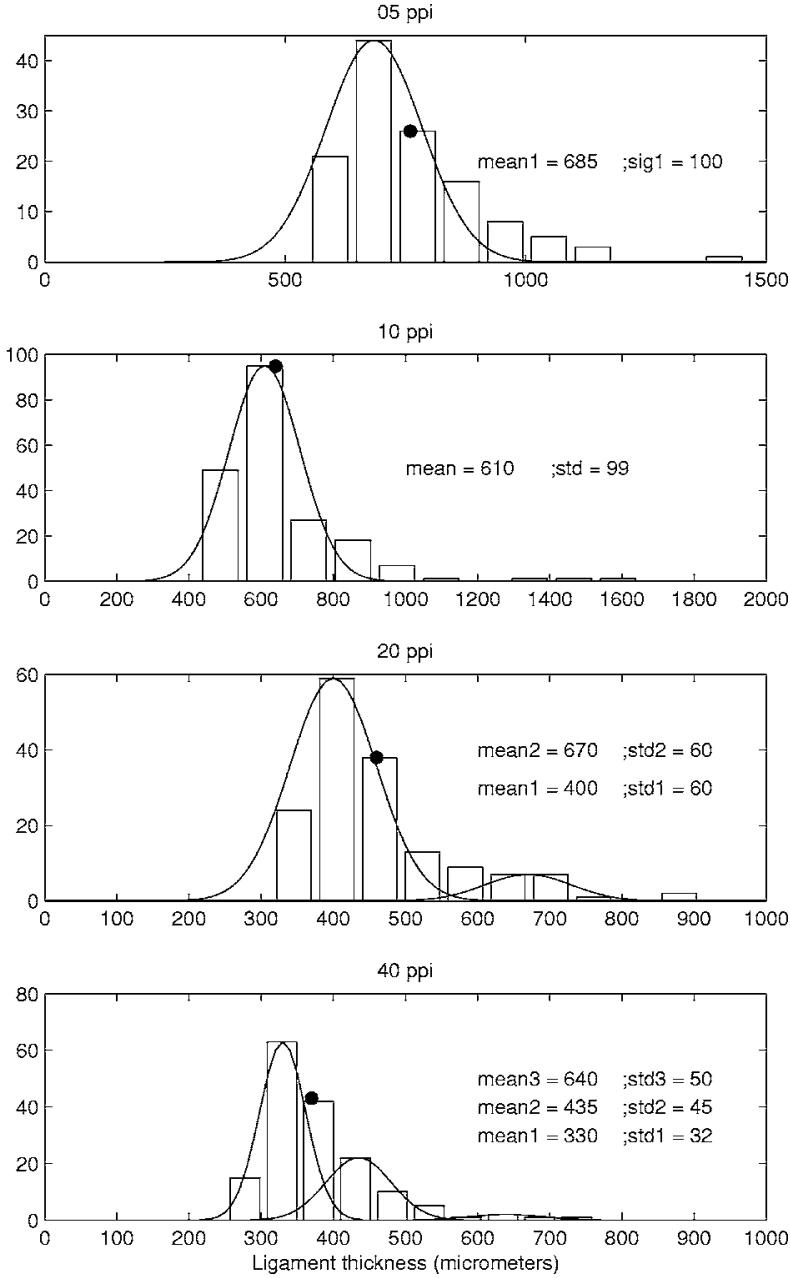

FIG. 5. Length and thickness distributions of the ligaments for the four ERG open-cell aluminum foams.

ral systems. ${ }^{7}$ These observations tend to promote an orthotropic model for the representative idealized periodic unit cell with a constant ligament thickness.

\section{B. Representativity and limitations}

In this section, we first investigate the representativity of the quantitative analysis with respect to the number of analyzed cells. As noted previously, when the cells are taken individually, the variation coefficients on the ligament length and thickness are relatively large, in the order of $30 \%$ and $20 \%$, respectively. On the other hand, if the statistics are performed on all the cells of a given material (here a given pore density), the variation coefficients now drastically drop to a small value $(\mathrm{VC}<7 \%)$. For a finer analysis, the $\mathrm{VC}$ of the principal characteristic radii, the ligament length, and the ligament thickness is plotted in function of the number of analyzed cells in Fig. 6. As expected, the VC is decreasing with the number of analyzed cells. In the particular case of the studied open-cell aluminum foams, these results tend to demonstrate the stability and reproducibility of the quantification with a small number of mean-size cells extracted from the reconstructed samples. The reduction in the number of analyzed cells allows one to minimize the measurement time at the expense of the bias introduced in the results (increase of the VC). In our case, the low number of analyzed cells is a trade-off between the time duration of the experimental protocol and accuracy in the searched parameters.

To go further in the representativity of our analysis, Fig. 7 compares the mean results given in Table III to results on ERG foams that can be found in the literature. The comparisons are in terms of ligament length, ${ }^{6}$ ligament thickness, ${ }^{6,8,9}$ and characteristic radii. ${ }^{6,8}$ It is worth noting that Schmierer et
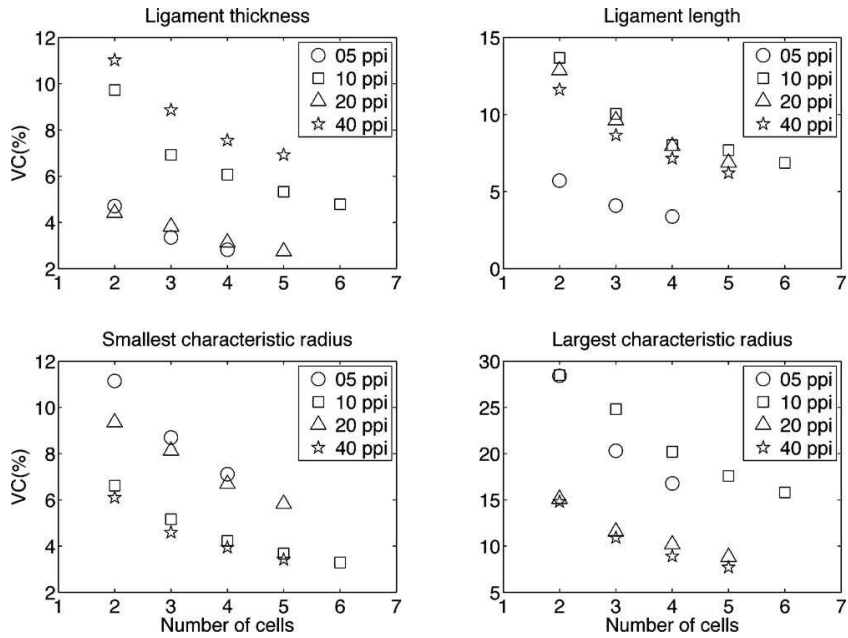

FIG. 6. Variation of cell size parameters with the number of cells (VC $=$ std $/$ mean) for the four ERG open-cell aluminum foams. 

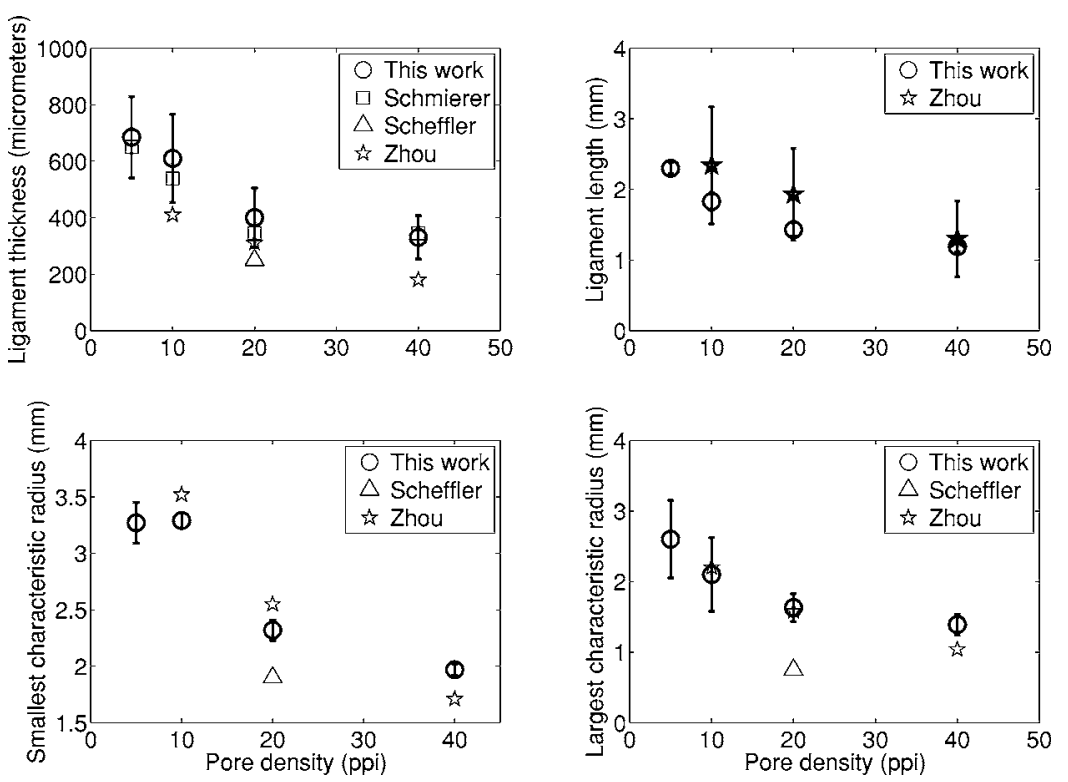

FIG. 7. Comparison of the cell size parameters measured on the three-dimensional reconstructed cells shown in Fig. 4 to results reported in the literature for the four ERG open-cell aluminum foams. $a l .{ }^{9}$ have measured the ligament hydraulic diameter $D_{h}$, whereas in Zhou et al. $^{6}$ and Scheffler et al. ${ }^{8}$ studies, as well as in the present one, a measure of the ligaments thickness is given by the height $h$ of a triangular cross section $\left(D_{h}\right.$ $=2 h / 3)$. For this reason, Schmierer et al.'s data have been multiplied by $3 / 2$ in order to be comparable to others. Once this conversion has been done, data from Schmierer et al. compare very well to those from Zhou et al., Scheffler et al., and ours.

While this method offers a new way of identifying the microstructural parameters of an open-cell foam, it has some limitations. First, it is relatively time consuming. In addition, current $\mathrm{x}$-ray $\mu \mathrm{CT}$ laboratory equipments have a limited resolution of about $5-10 \mu \mathrm{m}$. This precludes the analysis of typical sound absorbing foams like melamine with available $\mathrm{x}$-ray $\mu \mathrm{CT}$ laboratory equipments, whose ligament thickness is of the order of the minimal resolution. The use of synchrotron radiation $\mu \mathrm{CT}$, allowing the acquisition of high resolution three-dimensional images with a cubic voxel size less than $1 \mu \mathrm{m}$, is then required. ${ }^{22}$ It is also worth mentioning that, when such a tool is not available, quantitative results of the cellular morphology can be obtained by a destructive but accurate optical microscopy and stereological image analysis based method, ${ }^{6}$ which was found to give results in excellent agreement with those in the present article.

\section{Purely geometrical macroscopic parameters of reconstructed unit cells}

This paragraph briefly discusses comparison of Tables I and II. The upper bound of the overall experimental number of faces per cell $f$ is approximately 13.74. This is comparable to the mean number of faces per cell for the two main idealized unit cells presented in Table II. Considering now the statistics on the overall experimental number of edges per face, $n$ typically ranges from 5.02 to 5.26. This second topological parameter is still in good agreement with the main unit cells of Table II. Following this discussion, we may conclude that any one of the main unit cells of Table II could be proposed as a good candidate for representing the typical topology of the studied foams. For the sake of simplicity, a tetrakaidecahedron will be used in the following reconstruction process of the idealized PUC. Note that the tetrakaidecahedron is an approximation of the Kelvin cell.

Once the unit-cell model has been identified from the measured shape parameters given in Table I, some idealized PUCs can be built according to the different measured size parameters provided in Table III. Here, two reconstruction examples of a tetrakaidecahedral shaped unit cell with triangular ligament cross sections (cs) are presented for the $40 \mathrm{ppi}$ open-cell aluminum foam. First, an isotropic idealized PUC is simply reconstructed from the knowledge of the mean ligament length and thickness-see Fig. 8(a). Second, an orthotropic idealized PUC is reconstructed-see Fig. 8(b). This time the mean ligament thickness together with both the smallest characteristic radius and degree of anisotropy (or the largest characteristic radius) are required. For example, the mean ligament thickness, smallest characteristic radius, and degree of anisotropy of the 40-ppi foam are respectively $t$ $=0.37 \mathrm{~mm}, R_{1}=1.39 \mathrm{~mm}$, and DA=1.42 — see Tables III and IV. To reconstruct the orthotropic cell, an isotropic cell is first reconstructed using the first mode $l_{1}$ of the ligament length distribution. This length can be identified on Fig. 5 by mean 1, or approximated by the following geometrical relationship that holds for a tetrakaidecahedron unit cell: $R_{1}$

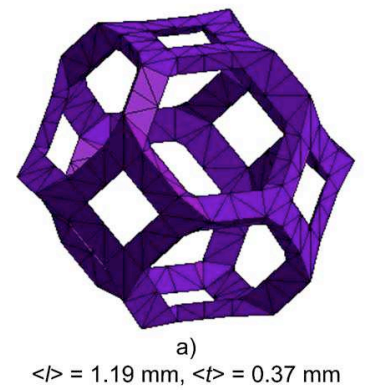

$\rightarrow \Phi=0.9462, \Lambda^{\prime}=2.4291 \mathrm{~mm}$

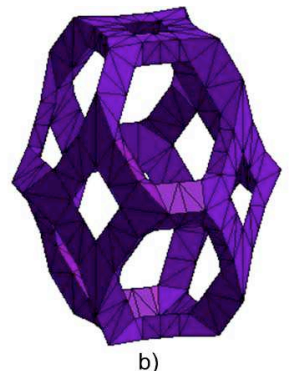

$R_{1}=1.39 \mathrm{~mm}, R_{3}=1.97 \mathrm{~mm},\langle t>=0.37 \mathrm{~mm}$ $\rightarrow \Phi=0.9228, \Lambda^{\prime}=1.8704 \mathrm{~mm}$
FIG. 8. Examples of reconstructed unit cells for the 40 ppi Duocel aluminum foam sample: (a) isotropic and (b) orthotropic unit cells. 
TABLE V. Open porosity $\Phi$ calculated on the reconstructed isotropic and orthotropic idealized unit cells compared to experimental measurements and literature data for the four ERG open-cell aluminum foams (mean \pm standard deviation).

\begin{tabular}{|c|c|c|c|c|}
\hline & $05 \mathrm{ppi}$ & $10 \mathrm{ppi}$ & $20 \mathrm{ppi}$ & $40 \mathrm{ppi}$ \\
\hline Reconstructed unit-cell: isotropic & $0.940 \pm 0.019$ & $0.932 \pm 0.027$ & $0.943 \pm 0.032$ & $0.946 \pm 0.041$ \\
\hline Reconstructed unit-cell: orthotropic & $0.908 \pm 0.028$ & $0.900 \pm 0.037$ & $0.915 \pm 0.045$ & $0.921 \pm 0.052$ \\
\hline Measurement (thermodynamic) & $0.915 \pm 0.001$ & $0.917 \pm 0.001$ & $0.914 \pm 0.001$ & $0.921 \pm 0.001$ \\
\hline Measurement (Archimedes) & $0.921 \pm 0.001$ & $0.922 \pm 0.001$ & $0.921 \pm 0.002$ & $0.926 \pm 0.002$ \\
\hline Measurement (by alloy density) & $0.918 \pm 0.002$ & $0.916 \pm 0.002$ & $0.915 \pm 0.001$ & $0.922 \pm 0.001$ \\
\hline TetraK circular cs & 0.909 & 0.898 & 0.914 & 0.919 \\
\hline TetraK triangular cs & 0.933 & 0.925 & 0.937 & 0.941 \\
\hline Zhou et al. ${ }^{\mathrm{a}}$ & & 0.935 & 0.932 & 0.918 \\
\hline Scheffler et $a l^{\mathrm{b}}$ & & & 0.922 & \\
\hline Schmierer et $a ._{.}^{\mathrm{c}}$ & 0.920 & 0.914 & 0.915 & 0.914 \\
\hline
\end{tabular}

${ }^{\mathrm{a}}$ Reference 6 .

${ }^{\mathrm{b}}$ Reference 8 .

${ }^{\mathrm{c}}$ Reference 9 .

$\approx l_{1} \sqrt{2}$. Consequently, an initial isotropic PUC having ligament length and thickness, respectively, equal to $l_{1}$ $=0.983 \mathrm{~mm}$ and $t=0.37 \mathrm{~mm}$ is reconstructed, and then elongated with a DA of 1.42 -see Fig. 8(b).

Finally, purely geometrical macroscopic parameters such as the open porosity $\Phi$ and the thermal characteristic length $\Lambda^{\prime}$ of the reconstructed idealized PUCs are simply obtained by spatial integration of elementary volume and surface elements. In Tables V and VI, the open porosity and the thermal characteristic length determined with the present reconstruction method of idealized unit-cells are compared to experimental and literature results. Note that the $\mu \mathrm{CT}$ measurement uncertainty of $87.2 \mu \mathrm{m}$ (Sec. III C) on the input values $l, t$, and $R_{1}$ were taken into account to evaluate the uncertainty on $\Phi$ and $\Lambda^{\prime}$.

\section{Open porosity}

In Table V, the open porosities $\Phi$ calculated on the idealized isotropic and orthotropic PUCs are compared to experimental measurements and literature data. Three experimental methods were used to measure the open porosity of the tested specimens. The first one is based on a thermodynamic method ${ }^{23}$ and the second on Archimedes principle. ${ }^{24}$ Since the density of the aluminum alloy is known, a third method is used to estimate this time the total porosity (open and closed cells) from the mass of the specimen and its bulk volume. If the specimen only contains open cells, open and total porosities are identical-this is what is observed in the results shown in Table V. As one can note, the orthotropic idealized PUC yields better comparisons with the measurements and literature results than the isotropic idealized PUC. To complete these comparisons, two other analytical methods are used: "TetraK circular cross section" and "TetraK triangular cross section." These methods are based on simple analytical equations derived in Appendix C, where the cell is considered isotropic. In this case, it is noted that the open porosity found for the triangular cross section is close to the one found for the isotropic idealized PUC. This is logical since the simple analytical equations assume an isotropic cell. While the analytical expressions can be used to obtain a rapid estimate of the porosity, they should be used with care since the analytical calculus overestimates the solid volume by counting several times the solid parts at the junctions, and assume an isotropic cell.

\section{Thermal characteristic length}

In Table VI, the thermal characteristic lengths $\Lambda^{\prime}$ calculated on the idealized isotropic and orthotropic PUCs are

TABLE VI. Thermal characteristic length $\Lambda^{\prime}(\mathrm{mm})$ calculated on the reconstructed isotropic and orthotropic idealized unit cells compared to experimental measurements and data reported in the literature for the four ERG open-cell aluminum foams. Parentheses indicate the relative difference when compared to the reconstructed orthotropic idealized unit-cell method.

\begin{tabular}{|c|c|c|c|c|}
\hline & 05 ppi & $10 \mathrm{ppi}$ & 20 ppi & 40 ppi \\
\hline Reconstructed unit-cell: isotropic & $4.45 \pm 1.07$ & $3.35 \pm 0.80$ & $2.84 \pm 0.98$ & $2.43 \pm 1.02$ \\
\hline Reconstructed unit-cell: orthotropic & $3.13 \pm 0.56$ & $2.56 \pm 0.57$ & $2.10 \pm 0.68$ & $1.85 \pm 0.76$ \\
\hline Acoustic characterization method & $2.67 \pm 0.01$ & $2.30 \pm 0.06$ & $1.93 \pm 0.23$ & $2.01 \pm 0.43$ \\
\hline Heat transfer experiments ${ }^{\mathrm{a}}$ & $3.12(-0.3 \%)$ & $2.63(3 \%)$ & $2.17(3 \%)$ & $1.79(-3 \%)$ \\
\hline Three-dimensional $\mu \mathrm{CT}^{\mathrm{b}}$ & $2.13(-32 \%)$ & $1.92(-25 \%)$ & $1.47(-30 \%)$ & $1.32(-29 \%)$ \\
\hline Two-dimensional $\mu \mathrm{CT}: P-A^{\mathrm{b}}$ & $2.63(-16 \%)$ & & $1.67(-20 \%)$ & \\
\hline TetraK circular cs and spherical nodes ${ }^{c}$ & $3.12(-0.3 \%)$ & $2.33(-9 \%)$ & $1.89(-10 \%)$ & $1.61(-13 \%)$ \\
\hline TetraK circular cs & $3.80(21 \%)$ & $2.82(9 \%)$ & $2.44(17 \%)$ & $2.11(12 \%)$ \\
\hline TetraK triangular cs & $3.54(13 \%)$ & $2.63(2 \%)$ & $2.27(8 \%)$ & $1.96(4 \%)$ \\
\hline
\end{tabular}

${ }^{\mathrm{a}}$ References 25-28.

${ }^{\mathrm{b}}$ Reference 9 .

${ }^{\mathrm{c}}$ Reference 29. 


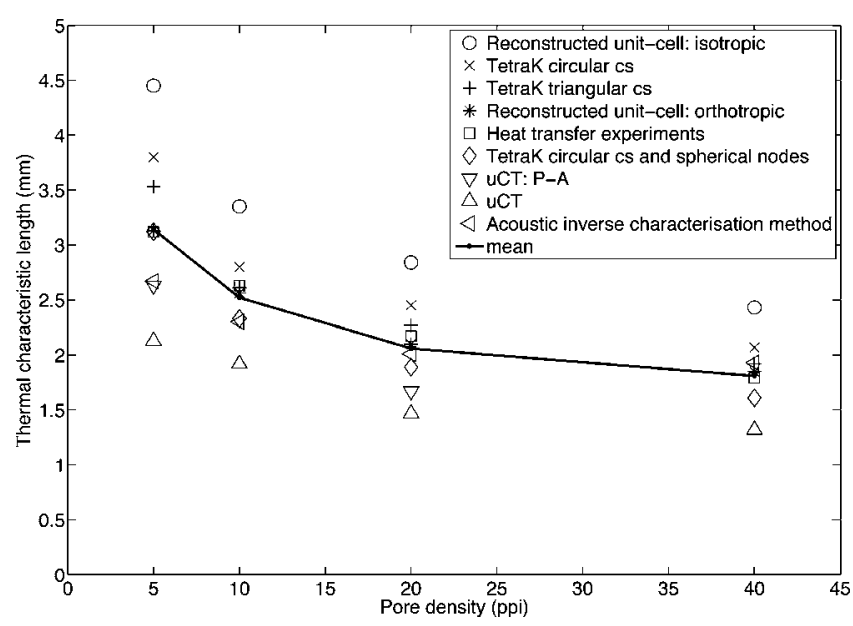

FIG. 9. Thermal characteristic length $\Lambda^{\prime}(\mathrm{mm})$ determined by reconstruction of an idealized unit cell and compared to data reported in the literature and acoustical measurements for the studied ERG open-cell aluminum foams of (a) 5, (b) 10, (c) 20, and (d) 40 ppi.

compared to experimental measurements and literature data. Here, the method used to measure $\Lambda^{\prime}$ is based on an inverse acoustical approach discussed elsewhere. ${ }^{30}$ As shown in Table VI, the orthotropic unit-cell reconstruction method is again leading to better comparisons with measurements and literature data than the isotropic unit cell. This is more clearly shown in Fig. 9, where all of the results of ERG open-cell aluminum foams are compared to the overall mean value per pore-density (thick line)_note that the mean value does not take into account the results of the orthotropic idealized PUCs to prevent bias. As one can note, the thermal characteristic length calculated using the orthotropic PUC falls on the mean value for each of the pore densities.

From the comparisons with the literature data, it is noted that our estimations of $\Lambda^{\prime}$ based on the orthotropic PUC are particularly very close to those deriving from mixed conduction/convection heat transfer experiments in open-cell metal foams, ${ }^{25-28}$ with a relative difference of the order of $3 \%$. On the contrary, literature data on $\Lambda^{\prime}$ obtained from direct integration of three-dimensional $\mu \mathrm{CT}$ images ${ }^{9}$ are giving large relative differences of the order of $-30 \%$. These relative differences may be due to $\mu \mathrm{CT}$ image artifacts, which introduce artificial roughness at the surface of the imaged samples (Sec. II C 3), reducing by this way the computed thermal characteristic length. This is confirmed by the fact that, when $\Lambda^{\prime}$ is obtained from serial integrations of fluid area $(A)$ to solid perimeter $(P)$ on two-dimensional $\mu \mathrm{CT}$ slices (referred as the " $\mu \mathrm{CT}: P-A$ " method), the relative difference is decreasing, because the weight of artificial surface roughness is becoming less important in two dimensions. We argue that the reconstruction approach of idealized periodic unit cells proposed in this article is correcting erroneous surface information originating from polychromatic $\mu \mathrm{CT}$ images, notably by the visual surface inspection compared to optical micrographs (Sec. II C 3). On the other hand, comparisons with the so-called "TetraK method" are leading to intermediate relative differences of the order of $8 \%$. In this last method, the metal foam structure is also assumed to be composed of a tetrakaidecahedral lattice, but this time the ligaments are chosen to be cylindrical in shape, with spherical nodes at the vertices. ${ }^{29}$ There exist experimental evidences that the cross section of the ligaments connecting the nodes is, in general, a function of the open porosity, and changes from circular (for $\Phi \approx 0.85$ ) to concave triangle (for $\Phi \approx 0.97$ ). ${ }^{25}$ Schmierer et $a .^{29}$ were aware of this property; nevertheless this cross-section variation with porosity was neglected in their model. Following this, since the studied samples have a measured open porosity of approximately 92\% (see Table IV), it is supposed that the ligament cross section should be better approximated by a triangular rather than circular shape. This explains the relative difference of approximately $8 \%$ mentioned earlier.

To complete these comparisons, the two analytical methods discussed previously and derived in Appendix $\mathrm{C}$ are used. In this case, relative differences of approximately $7 \%$ (for triangular cross section) and $15 \%$ (for circular cross section) are introduced and compared to $\Lambda^{\prime}$ values found from the orthotropic idealized PUCs. Once again, these analytical expressions can be used to provide rapid approximations of $\Lambda^{\prime}$; however, they should be used with care since by counting several times the solid volume at the junctions, the value found for $\Lambda^{\prime}$ is systematically overestimated.

\section{CONCLUDING REMARKS}

In this article, a cellular morphology characterization method for open-cell reticulated foams was presented. First, direct measurements of the cell shape and size parameters are performed at the cellular level on three-dimensional polychromatic x-ray $\mu \mathrm{CT}$ images. Second, from the measured local geometry parameters, a procedure has been proposed to reconstruct the cellular medium using the concept of an idealized PUC. While the procedure is lengthy, it is straightforward and does not use any adjustable parameters. The method has been successfully applied to the reconstruction of idealized PUCs for four high porosity open cell aluminum foams, where good agreements were obtained with experimental measurements and literature data in terms of the various cellular morphological and purely geometrical macroscopic parameters.

Perhaps the most outstanding result is the ability of the method to produce a PUC of a porous medium, which accurately renders the dependence between the purely geometrical macroscopic parameters (such as open porosity and thermal characteristic length) and the local geometry analyzed at the cell size level. Also, the method has proved to yield accurate evaluations of the macroscopic parameters that can be used to validate existing macroscopic characterization methods (e.g., ultrasound and inverse techniques). These macroscopic parameters are of utmost importance to characterize the thermal dissipation mechanisms in sound absorbing materials. $1,10,31$

Finally, the PUC approach is presented here as a simple microstructural method that could be used to extract other macroscopic and dynamic acoustical properties from a porous medium, such as the dynamic thermal and viscous permeabilities, directly computed on a three-dimensional reconstructed idealized PUC. ${ }^{32}$ 


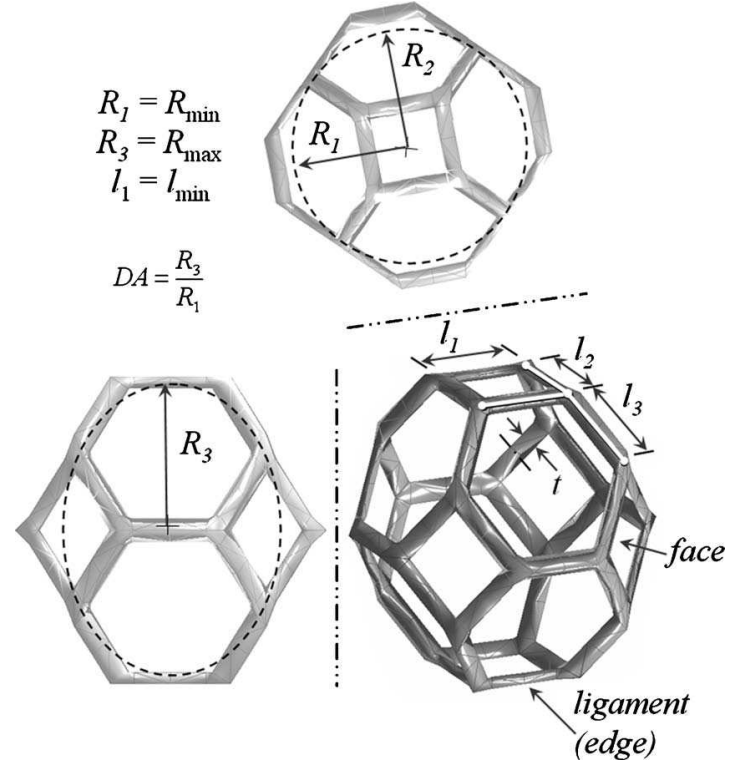

FIG. 10. The shape and size parameters measured on a unit cell.

\section{ACKNOWLEDGMENTS}

The authors wish to thank Marc Lefebvre and Richard Bouchard for their help in the characterization of $\mu \mathrm{CT}$ images. They also acknowledge the technical assistance provided by Irène Kelsey from the Institut des Matériaux et Systèmes Intelligents. This work was partially supported by Alcan, REGAL, CQRDA, and NSERC/CRD/GM Canada. The first author also acknowledges the academic grant provided by Région Rhône-Alpes.

\section{APPENDIX A}

The open porosity $\Phi$ of a porous solid is defined as the fraction of the interconnected pore fluid volume $V_{f}$ to the total bulk volume of the porous aggregate $\Omega$,

$$
\Phi=V_{f} / \Omega .
$$

The thermal characteristic length $\Lambda^{\prime}$, which is a generalization of the hydraulic radius, is equal to twice the interconnected pore fluid volume $V_{f}$ to the pore wet surface $\Gamma$ ratio

$$
\Lambda^{\prime}=2 V_{f} / \Gamma
$$

\section{APPENDIX B}

The shape and size parameters to measure on individual $\mu \mathrm{CT}$ reconstructed cells are depicted in Fig. 10. The faces are the windows bounded by ligaments-for open-cell foams, there is no wall on the faces. The corners of the faces are the vertices or nodes. The ligaments are the elongated elements linking two vertices. Three lengths of ligament are defined: the smallest or mode- 1 length $l_{1}$, the mode- 2 length $l_{2}$, and the largest or mode- 3 length $l_{3}$. The mean ligament length $l$ is the average of the three modes. For isotropic cells, the three lengths are equivalent, and typically, for orthotropic cells $l_{1}=l_{2} \neq l_{3}$, and for anisotropic cells $l_{1} \neq l_{2} \neq l_{3}$. Similarly, three radius modes exist: mode- 1 radius $R_{1}$, mode-2 radius $R_{2}$, and mode- 3 radius $R_{3}$. For anisotropic cells, mode- 1 and mode- 2 refer to the smallest and largest radius between $R_{1}$ and $R_{2}$, respectively. Here, the degree of anisotropy is then given by $\mathrm{DA}=R_{3} / R_{1}$. It should be noted that radius $R_{i}$ is mainly associated to length $l_{i}$. Finally, only one ligament thickness $t$ is defined per cell.

The measurements of the radii are performed by defining the largest ellipses that can be drawn interior to orthogonal planes of a cell-i.e., the ellipses are inscribed within the cell. Consequently, assuming orthotropic cells, only two radii per cell have to be measured. The length of a ligament is the distance between its extremity nodes, measured center to center, and its thickness is measured at the third of its length. For a tetrakaidecahedron cell, it means that a maximum of 36 lengths and 36 thicknesses can be measured per cell. The only one ligament thickness is obtained by averaging all 36 thicknesses.

\section{APPENDIX C}

The purpose of this appendix is to present simple analytical equations to estimate the volume and surface properties of open-cell foams. Gibson and Ashby ${ }^{1}$ have noticed that if the cell edge length is $l$ and the cell wall thickness is $t$, and $t \ll l$-that is the porosity $\Phi$ is high—-then for all open-cell foams

$$
\Phi=1-C\left(\frac{t}{l}\right)^{2},
$$

where $C$ is a numerical constant, near unity, that depends on the details of the cell shape. Assuming a tetrakaidecahedron unit-cell, this constant can be analytically calculated for different cross-section shapes. For the case of an equilateral triangular cross section of height $h(t=h), C=\sqrt{3} / 2 \sqrt{2}$; for a circular cross-section shape of diameter $d(t=d), \quad C$ $=3 \pi / 8 \sqrt{2}$. The thermal characteristic length $\Lambda^{\prime}$ can also be estimated from local measurements via a second equation, which takes the following form:

$$
\Lambda^{\prime}=D_{1} \frac{l^{2}}{t}-D_{2} t
$$

where $D_{1}$ and $D_{2}$ are still numerical constants near unity which can be determined analytically for simple cases. For an equilateral triangle cross-section shape of height $h$, we found $D_{1}=2 \sqrt{2} / 3 \sqrt{3}$, and $D_{2}=1 / 3$. For a circular crosssection shape of diameter $d, D_{1}=4 \sqrt{2} / 3 \pi$ and $D_{2}=1 / 2$.

${ }^{1}$ J. F. Allard, Propagation of Sound in Porous Media: Modeling Sound Absorbing Materials (Elsevier Applied Science, New York, 1993).

${ }^{2}$ T. J. Lu, A. Hess, and M. F. Ashby, J. Appl. Phys. 85, 7528 (1999).

${ }^{3}$ L. J. Gibson and M. F. Ahsby, Cellular Solids: Structure and Properties (Cambridge University Press, Cambridge, 1997).

${ }^{4}$ D. Weaire and S. Hutzler, The Physics of Foams (Oxford University Press, Oxford, 1999).

${ }^{5}$ K. A. Brakke, Exp. Math. 1, 141 (1992).

${ }^{6}$ J. Zhou, C. Mercer, and W. O. Soboyejo, Metall. Mater. Trans A 33, 1413 (2002).

${ }^{7}$ M. D. Montminy, A. R. Tannenbaum, and C. W. MacOsko, J. Colloid. Interface Sci. 280, 202 (2004).

${ }^{8}$ F. Scheffler, R. Herrmann, W. Schwieger, and M. Scheffler, Microporous Mesoporous Mater. 67, 53 (2004).

${ }^{9}$ E. N. Schmierer, A. Razani, T. Melton, and S. Keating, Proceedings of 
2004 ASME International Mechanical Engineering Congress and RD\&D Expo (ASME, New York, 2004).

${ }^{10}$ M. Henry, P. Lemarinier, J. F. Allard, J. L. Bonardet, and A. Gedeon, J. Appl. Phys. 77, 17 (1995).

${ }^{11}$ M. F. Ashby, A. Evans, N. A. Fleck, L. J. Gibson, J. W. Hutchinson, and H. N. G. Wadley, Metal Foams: A Design Guide (Elsevier ButterworthHeinemann, New York, 2000).

${ }^{12}$ T. G. Nieh, K. Higashi, and J. Wadsworth, Mater. Sci. Eng., A 283, 105 (2000).

${ }^{13}$ Y. Conde, J. F. Despois, R. Goodall, A. Marmottant, L. Salvo, L. San Marchi, and A. Mortensen, Adv. Eng. Mater. 8, 795 (2006).

${ }^{14}$ A. Feldkamp, L. C. Davis, and J. W. Kress, J. Opt. Soc. Am. A 1, 612 (1984).

${ }^{15}$ R. A. Ketcham and W. D. Carlson, Comput. Geosci. 27, 381 (2001).

${ }^{16}$ E. J. Garboczi, M. F. Thorpe, M. DeVries, and A. R. Day, Phys. Rev. A 43, 6473 (1991).

${ }^{17}$ K. R. Castleman, Digital Image Processing (Prentice-Hall, Englewood Cliffs, NJ, 1996).

${ }^{18}$ Sir William Thomson (Lord Kelvin), Philos. Acta Math. 11, 121 (1887).

${ }^{19}$ D. Weaire and R. Phelan, Philos. Mag. Lett. 69, 107 (1994).

${ }^{20}$ E. B. Matzke, Am. J. Bot. 33, 58 (1946).

${ }^{21}$ A. M. Kraynik, D. A. Reinelt, and F. van Swol, Phys. Rev. E 67, 031403
(2003).

${ }^{22}$ A. Elmoutaouakkil, G. Fuchs, P. Bergounhon, R. Peres, and F. J. Peyrin, Physica D. 36, A37 (2003).

${ }^{23}$ Y. Champoux, M. R. Stinson, and G. A. Daigle, J. Acoust. Soc. Am. 89, 910 (1991)

${ }^{24}$ R. Panneton and E. Gros, Acta Acust. Acust. 91, 342 (2005).

${ }^{25}$ A. Bhattacharya, V. V. Calmidi, and R. L. Mahajan, Int. J. Heat Mass Transfer 45, 1017 (2002).

${ }^{26}$ V. V. Calmidi and R. L. Mahajan, ASME Trans J. Heat Transfer 122, 557 (2000).

${ }^{27}$ M. S. Phanikumar and R. L. Mahajan, Int. J. Heat Mass Transfer 45, 3781 (2002).

${ }^{28}$ C. Y. Zhao, T. Kim, T. J. Lu, and H. P. Hodson, J. Thermophys. Heat Transfer 18, 309 (2004).

${ }^{29}$ E. N. Schmierer, A. Razani, J. W. Paquette, and K. J. Kim, Proceedings of 2004 ASME Heat Transfer/Fluids Engineering Summer Conference, 2004.

${ }^{30}$ Y. Atalla and R. Panneton, Can. Acoust. 33, 11 (2005).

${ }^{31}$ D. Lafarge, P. Lemarinier, J. F. Allard, and V. Tarnow, J. Acoust. Soc. Am. 102, 1995 (1997).

${ }^{32}$ C. Perrot, R. Panneton, and X. Olny, J. Acoust. Soc. Am. 120, 3145 (2006). 